\title{
Joint Action and Biochemichal Alteration in Egyptian Cotton Leafworm, Spodoptera littoralis (Boisd.) against four Insecticides from Different Groups Hendawi, M. Y. ${ }^{1}$; A. A. M. Shalaby ${ }^{1}$; W. M. H. Desuky ${ }^{2}$ and A. E. El-Morshedy ${ }^{2}$ \\ ${ }^{1}$ Plant Prot. Dept., Fac. Agric., Zagazig Univ., Egypt \\ ${ }^{2}$ Plant Prot. Res. Institute, ARC, Dokki, Giza, Egypt
}

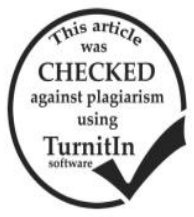

\begin{abstract}
Joint action and biochemical alteration in the $4^{\text {th }}$ instar larvae of a laboratory strain of cotton leafworm, Spodoptera littoralis (Boisd.), were evaluated under the laboratory condition against four insecticides from different groups namely: chlorpyrifos, chlorfluazuron, emamectin benzoate, pyrethrins. The toxicity effect for each single compound and binary mixture at levels 1:1, 1:2: and 2:1 from $\mathrm{LC}_{25}$ were evaluated. The study indicated that emamectin benzoate followed by chlorfluazuron proved to be the most effective compounds among all tested insecticides. The highest initial effect $(76.66 \%)$ was obtained by mixing chlorpyrifos: chlorfluazuron at level 1:2, respectively. while the lowest initial effect $(3.33 \%)$ was obtained as a result of mixing emamectin benzoate with pyrethrins at level 1:2, respectively, the same effect occurred with chlorpyrifos: pyrethrins at level 1:1. Changing in enzymatic activities as responses of treatment with mixtures at $\mathrm{LC}_{25}$ of all tested compounds, except for pyrethrins was studied at the recommended concentration, after 1and 3 days post treatment. Our study noticed that mixing of pesticides caused antagonism effect except chlorpyrifos plus pyrethrins at the ratio 2:1 caused potentiation effect. Under the effect of the tested insecticides the data showed increasing in total soluble protein and total lipids contents, reduction in carbohydrate hydrolyzing enzymes (amylase, invertase and trehalase), increasing in transaminase enzymes (AST and ALT) and reduction in phosphatase enzymes (ACP and ALP) activities .Our data recommend not to mix these insecticides due to the antagonistic effect.
\end{abstract}

Keywords: joint action, Spodoptera littoralis, enzymatic activity, pesticides, potentiation, antagonism

\section{INTRODUCTION}

Cotton leafworm Spodoptera littoralis (Boisd.) is one of the most damaging phytophagous insect pests in Egypt, larvae of this pest can attack not only to cotton but also to other field crops and vegetables and the devolpment and growth rate has strong nutritional relations (Abd ElMageed and Shalaby, 2011; Metayi et al., 2015).

Because of extensively use of chemical insecticides during the last few years, Egypt is facing a big problem from the resistance devolpment in cotton leafworm (ElBermawy et al., 1992; Rashwan et al., 1992).

Find alternative ways to solve the problem of resistance in future had led to investigating it in mixtures, to improve their performance and elongate their life as effective control measures. Insecticeds mixtures involve combinations of two or more are broadly used to deal with a range of arthropod pests in greenhouse and open fields. Moreover, the use of pesticide mixtures may lead to synergism or potentiation and the reduction of resistance (Tabashnik, 1989 and Ahmad, 2009).

Mixing of chemicals of different mode of action induce the potent use of toxicants (synergism) which could hypothetically prevent or interruption the emergence of resistant strains. In this regard, it was reported that organophosphates synergize/pyrethroids against several pests, i.e., S. littoralis (Temerak, 2002 and Ascher et al., 1986), also, synergism was acquired by combinations of IGRs and traditional insecticides (El-Guindy et al., 1985; Radwan et al., 2009 and Kandil et al., 2006).

However, antagonism may also happen because of mixing two (or more) pesticides together. Care should be taken when integrated pesticide mixtures with biological control agents is especially important because parasitoids and predators can suppress arthropod pest populations irrespective of the arthropod pests' resistance traits or mechanisms (Tabashnik, 1986 and Ascher et al., 1986).

Though, the present investigation aimed to study and characterize the joint action between four insecticides from different groups namely: chlorfluazuron, emamectin benzoate and pyrethrins compared to one of the most known organophosphorous compound (chlorpyrifos) as a chemical insecticide, when tested against the $4^{\text {th }}$ instar larvae of the cotton leafworm S. littoralis (Biosd.).

\section{MATERIALS AND METHODS}

\section{1-Compounds Tested}

1- Chlorpyrifos (Dursban 48\% EC) an organophosphorous compound. Product (1 L/ fad.) supplied by Dow Agro Sciences company.

2- Chlorfluazuron (Caprice 5\% EC) IGRs' compound. Product (400 ml/fad.) supplied by Elhelb pesticides company.

3- Emamectin benzoate (Pasha 1.9\% EC) bio-insecticide. Product $(250 \mathrm{ml} / \mathrm{fad}$.) supplied by Elhelb pesticides and chemicals company.

4- Pyrethrins (Pyrethrum 5\% EC) a plant extract. Product (440 $\mathrm{ml} /$ fad.) supplied by Agropharm Ltd (UK) company.

The formulated samples of the tested insecticides were obtained from Plant Protection Research Institute (PPRI), Agricultural Research Centre (ARC), Dokki, Giza. 2-Test insects:

A susceptible strain of $S$. littoralis (Boisd.) was obtained from Agricultural Research Centre (ARC), Dokki, Giza. Tested insects were reared and tested under constant conditions $26 \pm 1^{\circ} \mathrm{C}$ and $65 \pm 5 \% \quad \mathrm{RH}$. and photoperiod 12:12 L: D as described by El-Defrawi, et al. (1964). The experiments were carried out in the Laboratory of Biological Control, Plant Protection Research Institute, Sharkia Branch.

3. - LC values determination for the tested compounds

For assessing the toxicity of each insecticide either separately or in binary mixtures, stock solution of each insecticide alone or mixture of two compounds were 
prepared fresh daily based on active ingredient (w/v). Six deffrent concentrations for each compound were prepared freshly form stock concentration. The selected concentrations of each compound caused mortality from $20-80 \%$.

Leaf dipping technique was used to estimate the larvaicidal action of the tested insecticides and joint action against one-day-old $4^{\text {th }}$ instar larvae of $S$. littoralis. Each concentration was replicated three times, each replicate contained 10 lravae. Control experiments involved using leaf disks dipped in water. This trial was carried out in the incubator at $26 \pm 1^{\circ} \mathrm{C}$ and $65 \pm 5 \% \mathrm{RH}$. Larval mortality was scored: if no movement was observed, larvae were considered as dead after $24 \mathrm{hr}$. for chlorpyrifos and $72 \mathrm{hr}$. for the remaining insecticides.

Mortality counts were recorded; $\mathrm{LC}_{50}, \mathrm{LC}_{90}$, slope, toxicity index and relative potency values were calculated.

Toxicity index (T.I.) was determined by using Sun's equation (1950) as follow:

$$
\text { Toxicity index }=\frac{\mathrm{LC}_{50} \text { or } \mathrm{LC}_{90} \text { of the highest efficient compound }}{\mathrm{LC}_{50} \text { or } L C_{90} \text { of the other compound }}
$$

Relative potency (R.P.) values were measured according to the method described by Zidan and AbdelMegeed (1988).

Relative potency (fold) $=\frac{\mathrm{LC}_{50} \text { or } L C_{90} \text { of the lowest efficient compound }}{\mathrm{LC}_{50} \text { or } L C_{90} \text { of the other compound }}$

The joint action between tested insecticides at LC25 were prepared by mixing two insecticides in each combination with each other at three rates namely; LC25 of both insecticides (1:1), LC25 of one toxicant plus double of the LC25 of the LC25 of the other toxicant (1:2) and the reverse of the latter case $(2: 1)$ except for pyrethrins which used at recommended rate. At the same time samples of survived larvae were taken to determine the total soluble protein, total lipids, activities of aspartate aminotransferase (AST), alanine aminotransferase (ALT), carbohydrate hydrolyzing enzymes and phosphatase enzymes (ALP \& ACP) after 1 and 3 days of treatment.

\section{4- Biochemical assay.}

Samples Preparation for biochemical assay:

Larval samples used for biochemical assays were collected at 1 and 3 days post treatment of the $4^{\text {th }}$ instar larvae during the joint action trials. Untreated larvae were used as control. Samples were homogenized in distilled water $(50 \mathrm{mg} / \mathrm{ml})$ using chilled glass Teflon homogenizer. Homogenates centrifuged at 5000rpm for 20 min. at $5^{\circ} \mathrm{C}$ in a refrigerated centrifuge. The deposits were discarded and the supernatants were kept in a deep freezer at $-20{ }^{\circ} \mathrm{C}$ till used to determine the total soluble protein, the activities of aspartate aminotransferase (AST) and alanine aminotransferase (ALT), carbohydrate hydrolyzing enzymes (invertase, trehalase and amylase) and phosphatase enzymes (ALP \& ACP).

\section{Colorimetric determination}

Total soluble protein in supernatants of homogenate treated larvae of $S$. littoralis were carried out as described by Gornall, et al. (1949), The total lipids estimated with the method of Schmit (1964), Aspartate aminotransferase (AST) and Alanine aminotransferase (ALT) were determined colorimetrically according to the method of Reitman and Frankle (1957). The method used to determine the digestion of trehalose, starch and sucrose by trehalase (EC 3.2.1.28), amylase (EC 3.2.1.1) and invertase (EC 3.2.1.26) enzymes respectively, were similar to those described by Ishaaya and Swiriski (1976), acid phosphatase (EC 3.1.3.2) and alkaline phosphatase (EC 3.1.3.1) were accomplished by using P-nitrophenyl phosphate as a reaction substrate (Guilabault, 1970).

5-Statistical analysis:

All the obtained data were statistically analyzed according to (Tukey's HSD 1949). Data were subjected to statistical analyses using a software package Costat ${ }^{\circledR}$ Statistical Software (2005).

\section{RESULTS AND DISCUSSION}

\section{Tested compounds LC values}

The toxic effects were listed after $72 \mathrm{hr}$. for all treatments except chlorpyrifos was listed after $24 \mathrm{hr}$. due to its mode of action.

Data given in Table (1) show that the order of efficiency of the tested insecticides against $4^{\text {th }}$ instar larvae was the same at both the $\mathrm{LC}_{50}$ and $\mathrm{LC}_{90}$ levels. At the both mentioned levels, emamectin benzoate was the most potent insecticide, whereas, chlorpyrifos was the least effective one. The insecticide chlorfluazuron occupied an intermediate position. Korrat, et al. (2012) found that emamectin benzoate was the most effective insecticide $\left(\mathrm{LC}_{50}=0.017 \mathrm{ppm}\right)$ among all tested insecticides (chlorfluazuron, profenfos and spinosad).

Table 1. Acute toxicity of chlorpyrifos, chlorfluazuron and emamectin benzoate on $4^{\text {th }}$ instar larvae of $S$. littoralis at $26 \pm 1^{\circ} \mathrm{C}$ and $65 \pm 5 \%$ RH.

\begin{tabular}{|c|c|c|c|c|c|c|c|c|}
\hline \multirow{2}{*}{ Insecticides } & \multirow{2}{*}{$\begin{array}{l}\mathrm{LC}_{50} \\
\text { ppm }\end{array}$} & \multirow{2}{*}{$\begin{array}{l}\mathbf{L C}_{90} \\
\text { ppm }\end{array}$} & \multirow{2}{*}{$\begin{array}{l}\text { Slope } \\
\pm \text { SE }\end{array}$} & \multicolumn{2}{|c|}{ Toxicity index at } & \multicolumn{2}{|c|}{ Relative potency fold at } & \multirow{2}{*}{$\begin{array}{l}\mathbf{L C}_{90} / \\
\mathbf{L C}_{\mathbf{5 0}}\end{array}$} \\
\hline & & & & $\mathrm{LC}_{\mathbf{5 0}}$ & $\mathrm{LC}_{90}$ & $\mathbf{L C}_{50}$ & $\mathbf{L C}_{90}$ & \\
\hline Chlorpyrifos & 34.62 & 96.49 & $0.44 \pm 3.39$ & 0.21 & 0.17 & 1.00 & 1.00 & 2.79 \\
\hline Chlorfluazuron & 2.63 & 37.04 & $0.99 \pm 0.87$ & 2.82 & 0.44 & 13.17 & 2.60 & 14.08 \\
\hline Emamectin benzoate & 0.074 & 0.162 & $0.80 \pm 0.006$ & 100.00 & 100.00 & 467.81 & 595.62 & 2.19 \\
\hline
\end{tabular}

Toxicity Index and Relative Potency based on $\mathrm{LC}_{50}$

It seems always convenient to consider the efficiency or the degree of toxicity of different insecticides by comparing them with a standard compound. To achieve this, both the toxicity index method developed by Sun (1950), and the potency levels method (Zidan and Abdel-Megeed, 1988), frequently used in this respect were employed.
Therefore, emamectin benzoate and chlorpyrifos were considered the standars chemicals in calculating the toxicity index and potency levels, respectively.

Both of the toxicity index and potency levels data for the tested instars of S. littoralis at LC50 and LC90 levels are presented in Table (1). The results obtained revealed the general similarity in the trend of both the 
toxicity index and number of folds at both LC50 and LC90.

At the $\mathrm{LC}_{50}$ level, emamectin benzoate was the most toxic compound for the $4^{\text {th }}$ larvae of $S$. littoralis. The other two toxicants, i.e., chlorpyrifos and chlorfluazuron were 0.21 and $2.82 \%$ for the $4^{\text {th }}$ instar as toxic as emamectin benzoate, respectively.

At $\mathrm{LC}_{90}$, chlorpyrifos and chlorfluazuron were 0.17 and $0.44 \%$ as toxic as emamectin benzoate respectively. On ground of the number of folds, compared with chlorpyrifos, chlorfcluazuron and emamectin benzoate indicated 13.17, 467.81and 2.60 and 595.62 times as toxic as chlorpyrifos.

Regarding the slope values data given in Table (1) it is clear that a switch in position between chlorfluazuron and emamectin benzoate. Slope values of regression lines revealed that the larvae of S. littoralis were more homogeneity in their susceptibility to the tested toxicants. According to the estimated LC50 and LC90, it could be stated that the fourth instar reflected moderate level of susceptibility towards all the tested insecticides.
Concerning the toxicity of pyrethrins to the larvae of $S$. littoralis, it was found that the higher concentrations (recommended concentrations $2.2 \mathrm{ml} / \mathrm{L}$, respectively) gave mortality percentage did not exceed $20 \%$ after the third day of treatment.

For the joint action at LC25 level Data given in Table (2) show the observed percent mortality of tested compounds at LC25 level as well as at the double of the LC25, the observed percent mortality of binary mixtures at levels 1:1, 1:2 and 2:1 and co-toxicity factors (Co. f). Co-toxicity factor was calculated according to Mansour, et al. (1966).

With referace to the LC25 and LC50 values (Table 2) emamectin benzoate was the most effective insecticide that recorded $30.00 \%$. Meantime, chlorpyrifos and chlorfluazuron appeared to have a moderate effect recording 23.33 and $26.67 \%$ mortality, respectively. While, pyrethrins didn't cause any death at the recommended concentration .Data in the same table clearly show that when pyrethrins used at double of the LC25 (i.e. double of the recommended concentration) recorded $13.33 \%$ mortality.

Table 2. Toxic effects of the tested insecticides combination against the 4th instar larvae of $S$. littoralis at 1:1, 1:2 and 2:1 level using concentration LC25 with the exception of pyrethrins (recommended concentration).

\begin{tabular}{|c|c|c|c|c|c|c|c|c|c|c|c|c|c|}
\hline \multicolumn{2}{|c|}{ Compound } & \multicolumn{2}{|c|}{$\mathrm{LC}_{25}(\mathrm{ppm})$} & \multicolumn{4}{|c|}{$\begin{array}{cc}\begin{array}{c}\text { Observed percent } \\
\text { Observed percent } \\
\text { mortality at } \mathrm{LC}_{25} \\
\text { level }\end{array} & \begin{array}{l}\text { mortality at } \\
\text { double dose }\end{array} \\
\end{array}$} & \multicolumn{3}{|c|}{$\begin{array}{c}\text { Observed percent } \\
\text { mortality of } \\
\text { mixtures }\end{array}$} & \multicolumn{3}{|c|}{$\begin{array}{l}\text { Co-toxicity } \\
\text { factors }\end{array}$} \\
\hline $\mathbf{A}$ & B & $\mathbf{A}$ & B & $\mathbf{A}$ & B & $\mathbf{A}$ & B & $\begin{array}{cc}A & \text { B } \\
1: & 1\end{array}$ & $\begin{array}{cc}A & B \\
1: 2\end{array}$ & $\begin{array}{cc}\mathbf{A} & \mathbf{B} \\
2: & 1\end{array}$ & $\begin{array}{l}A \quad B \\
1: 1\end{array}$ & $\begin{array}{ll}A & B \\
1: 2\end{array}$ & $\begin{array}{ll}\text { A } & \text { B } \\
2: & 1\end{array}$ \\
\hline chlorpyrifos & chlorflus & 20.1 & 6537 & & 26. & $f^{\prime}$ & 53.33 & 30.00 & 76.66 & 30.00 & -40.00 & 1.23 & -59.09 \\
\hline & E. benzoate & 20.1896 & 0489 & & 30.00 & 46.6 & 56.66 & 6.67 & 20.00 & 53.33 & -87.49 & -75.00 & -30.44 \\
\hline " " " & pyrethrins & 20.1896 & Recom. & 23.33 & 0.00 & 46.67 & 13.33 & 3.33 & 6.67 & 60.00 & -85.73 & -81.81 & 28.56 \\
\hline chlorfluazuron & E. benzoate & 0.6537 & 0.0489 & 26.67 & 30.00 & 53.33 & 56.66 & 43.33 & 50.00 & 46.67 & -23.54 & -40.00 & -43.99 \\
\hline & pyrethrins & 0.6537 & Recom. & 26.67 & 0.00 & 53.33 & 13.33 & 30.00 & 13.33 & 60.00 & 12.49 & -66.68 & 12.51 \\
\hline E. benzoate & pyrethrins & 0.0489 & Recom. & 30.00 & 0.00 & 56.66 & 13.33 & 6.67 & 3.33 & 16.67 & -77.77 & -92.31 & -70.58 \\
\hline
\end{tabular}

observed percent mortality - expected percent mortality

Co-toxicity factor $=$

$$
\text { expected percent mortality }
$$

Co-toxicity factor $>\mathbf{2 0}$ means potentiation effect.

Co-toxicity factor $<-20$ means antagonistic effect.

Co-toxicity factor ranged between -20:20 means additive effect

Among the 36 binary mixtures strong enough to note that one mixture revealed potentiation (co.f. 28.56), this mixture contains op compound, chlorpyrifos + pyrethrins (2:1). Three cases of additive response, chlorpyrifos + chlorfluazuron (1:2); chlorfluazuron + pyrethrins $1: 1$ and $2: 1$. While the rest combinations revealed antagonistic effects and reduced the percent mortality when using them individually. The highest antagonistic effect (-92.31) was recorded when using emamectin benzoate + pyrethrins at level 1:2.

These results are in harmony with those obtained by Abdel-Megeed, et al. (2000), they revealed that chitin synthesis inhibitors were more potent when applied alone compared to their binary mixtures against the 4th instar larvae of S. littoralis when tested tebufenozide, benzoylphenylurea, chlorfluazuron, flufenoxuron, cutabron (profenofos + chlorfluazuron), Empire 50\% [chlorpyrifos+diflubenzuron] and AIMX (profenofos + chlorfluazuron). Shonouda, et al. (2000) tested the joint action of myrrh applied in binary mixtures with chemical insecticide (profenofos, chlorfluazuron, fenvalerate and pyriproxyfen) on larvae of S. littoralis. Results of the bioassay of mixtures indicated antagonistic effects on larval mortality. Khidr, et al. (2003) reported that higher reductions were observed in the number of larvae when chlorpyrifos was used in combination with chitin synthesis inhibitors.

Ghoneim, et al. (2012) mentioned that chlorpyrifos when mixed with chlorfluazuron produced additive effect based on co-toxicity factor against 4th instar larvae of S. littoralis. El-Sheikh (2015) illustrated that the combined effect of emamectin benzoate and lufenuron or spinosad was either additive or antagonistic; suggesting that single application of these insecticides is more effective than in combination.

\section{Biochemical responses}

Mixtures effects of chlorpyrifos, chlorfluazuron, emamectin benzoate and pyrethrins in at levels 1:1, 1:2 and 2:1of the LC25 on the levels of total soluble protein and total lipids as well as the activities of some enzymes in larval supernatants of 4 th instar larvae of $\mathrm{S}$. littoralis are shown in Tables (3 to 6). 
The total soluble protein:

Data in Table (3) showed the concentration of total soluble protein (TSP) in the larval supernatants of the 4th larval instar of the cotton leafworm, S. littoralis treated with the tested compounds in combinations. Generally, $50 \%$ of the treatments increased the total soluble protein levels comparing to control. The highest significant increase $(11.271 \pm 0.006 \mathrm{mg}$ protein / g body weight) after 1 day of treatment took place in case of emamectin benzoate : pyrethrins mixture at level 1:1 indicating $289.73 \%$, while after 3 days of treatment the highest significant increase, $9.120 \pm 0.005 \mathrm{mg}$ protein / g body weight $(88.27 \%)$, was obtained when using chlorfluazuron : chlorpyrifos at level 1:2. On the other hand, treatment of chlorpyrifos: emamectin benzoate (2:1) and chlorfluazuron: pyrethrins (1:1) recorded the highest significant decrease in the total soluble protein level after one and three days of treatment, respectively. The corresponding reduction percentages of protein level were -84.72 and $-55.64 \%$.

Table 3. Changes of total soluble protein and total lipid levels in $4^{\text {th }}$ larval instars of $S$. littoralis treated with the tested compounds' mixture.

\begin{tabular}{|c|c|c|c|c|c|}
\hline \multirow{2}{*}{ Treatment } & & \multicolumn{2}{|c|}{ Total soluble protein $* * *$} & \multicolumn{2}{|c|}{ Total lipids $* * * *$} \\
\hline & & 1 day & 3 days & 1 day & 3 days \\
\hline$\overline{\text { Control }}$ & Conc.* & $2.892 \pm 0.004 \mathrm{j}$ & $4.844 \pm 0.004 \mathrm{j}$ & $2.332 \pm 0.003 \mathrm{k}$ & $1.126 \pm 0.003 \mathrm{~m}$ \\
\hline Chlorflu. + Chlorpyr. & Conc. & $1.128 \pm 0.004 p$ & $3.458 \pm 0.004 \mathrm{~m}$ & $6.784 \pm 0.004 \mathrm{a}$ & $0.884 \pm 0.004 \mathrm{o}$ \\
\hline$(1: 1)$ & $\mathrm{C} \% * *$ & -61.00 & -28.61 & 190.91 & -21.49 \\
\hline $\begin{array}{l}\text { Chlorflu. + E. benzoate } \\
(1: 1)\end{array}$ & $\begin{array}{l}\text { Conc. } \\
\mathrm{C} \%\end{array}$ & $\begin{array}{c}7.143 \pm 0.004 \mathrm{c} \\
147.00\end{array}$ & $\begin{array}{c}7.986 \pm 0.004 \mathrm{e} \\
64.86\end{array}$ & $\begin{array}{c}1.339 \pm 0.004 \mathrm{o} \\
-42.58\end{array}$ & $\begin{array}{c}2.539 \pm 0.003 \mathrm{~g} \\
125.49\end{array}$ \\
\hline $\begin{array}{l}\text { Chlorflu. + Pyreth. } \\
(1: 1)\end{array}$ & $\begin{array}{l}\text { Conc. } \\
\mathrm{C} \%\end{array}$ & $\begin{array}{c}2.665 \pm 0.004 \mathrm{k} \\
-7.85\end{array}$ & $\begin{array}{c}2.149 \pm 0.004 \mathrm{~s} \\
-55.64\end{array}$ & $\begin{array}{c}5.847 \pm 0.003 \mathrm{c} \\
150.73\end{array}$ & $\begin{array}{c}3.875 \pm 0.003 b \\
244.14\end{array}$ \\
\hline $\begin{array}{l}\text { Chlorpyr. + E. benzoate } \\
(1: 1)\end{array}$ & $\begin{array}{l}\text { Conc. } \\
\mathrm{C} \%\end{array}$ & $\begin{array}{c}2.661 \pm 0.004 \mathrm{k} \\
-7.99\end{array}$ & $\begin{array}{c}3.179 \pm 0.004 n \\
-34.37\end{array}$ & $\begin{array}{c}4.364 \pm 0.004 \mathrm{~d} \\
44.25\end{array}$ & $\begin{array}{c}0.514 \pm 0.004 q \\
-54.35\end{array}$ \\
\hline $\begin{array}{l}\text { Chlorpyr. + Pyreth. } \\
(1: 1)\end{array}$ & $\begin{array}{l}\text { Conc. } \\
\mathrm{C} \%\end{array}$ & $\begin{array}{c}7.379 \pm 0.003 b \\
155.15\end{array}$ & $\begin{array}{c}6.506 \pm 0.004 \mathrm{~g} \\
34.31\end{array}$ & $\begin{array}{c}2.015 \pm 0.004 \mathrm{~m} \\
-13.59\end{array}$ & $\begin{array}{c}3.298 \pm 0.005 \mathrm{~d} \\
192.90\end{array}$ \\
\hline $\begin{array}{l}\text { E. benzoate + Pyreth. } \\
(1: 1)\end{array}$ & $\begin{array}{l}\text { Conc. } \\
\mathrm{C} \%\end{array}$ & $\begin{array}{c}11.271 \pm 0.006 \mathrm{a} \\
289.73\end{array}$ & $\begin{array}{c}6.979 \pm 0.004 \mathrm{f} \\
44.08\end{array}$ & $\begin{array}{c}2.543 \pm 0.004 \mathrm{j} \\
9.05\end{array}$ & $\begin{array}{c}2.955 \pm 0.004 \mathrm{e} \\
162.43\end{array}$ \\
\hline $\begin{array}{l}\text { Chlorflu. + Chlorpyr. } \\
(1: 2)\end{array}$ & $\begin{array}{l}\text { Conc. } \\
\mathrm{C} \%\end{array}$ & $\begin{array}{c}2.791 \pm 0.005 j \\
-3.49\end{array}$ & $\begin{array}{c}9.120 \pm 0.005 \mathrm{a} \\
88.27\end{array}$ & $\begin{array}{c}0.405 \pm 0.005 q \\
-82.63\end{array}$ & $\begin{array}{c}1.715 \pm 0.004 \mathrm{j} \\
52.31\end{array}$ \\
\hline $\begin{array}{l}\text { Chlorflu.+ E. benzoate } \\
(1: 2)\end{array}$ & $\begin{array}{l}\text { Conc. } \\
\mathrm{C} \%\end{array}$ & $\begin{array}{c}5.197 \pm 0.003 \mathrm{e} \\
79.70\end{array}$ & $\begin{array}{c}8.830 \pm 0.004 \mathrm{~b} \\
82.29\end{array}$ & $\begin{array}{c}2.139 \pm 0.0211 \\
-8.28\end{array}$ & $\begin{array}{c}2.329 \pm 0.004 \mathrm{~h} \\
106.84\end{array}$ \\
\hline $\begin{array}{l}\text { Chlorflu. + Pyreth. } \\
(1: 2)\end{array}$ & $\begin{array}{l}\text { Conc. } \\
\text { C\% }\end{array}$ & $\begin{array}{c}1.194 \pm 0.004 \mathrm{o} \\
-58.71\end{array}$ & $\begin{array}{c}3.124 \pm 0.004 \mathrm{o} \\
-35.51\end{array}$ & $\begin{array}{c}1.398 \pm 0.003 \mathrm{n} \\
-40.05\end{array}$ & $\begin{array}{c}4.695 \pm 0.004 a \\
316.96\end{array}$ \\
\hline $\begin{array}{l}\text { Chlorpyr. + E. benzoate } \\
(1: 2)\end{array}$ & $\begin{array}{l}\text { Conc. } \\
\mathrm{C} \%\end{array}$ & $\begin{array}{c}1.963 \pm 0.004 \mathrm{~m} \\
-32.12\end{array}$ & $\begin{array}{c}2.526 \pm 0.004 q \\
-47.85\end{array}$ & $\begin{array}{c}6.283 \pm 0.004 b \\
169.43\end{array}$ & $\begin{array}{c}0.845 \pm 0.004 p \\
-24.96\end{array}$ \\
\hline $\begin{array}{l}\text { Chlorpyr. + Pyreth. } \\
(1: 2)\end{array}$ & $\begin{array}{l}\text { Conc. } \\
\mathrm{C} \%\end{array}$ & $\begin{array}{c}4.691 \pm 0.004 \mathrm{~g} \\
62.21\end{array}$ & $\begin{array}{c}4.989 \pm 0.003 \mathrm{i} \\
3.00\end{array}$ & $\begin{array}{c}0.333 \pm 0.004 \mathrm{r} \\
\quad-85.72\end{array}$ & $\begin{array}{c}0.967 \pm 0.004 n \\
-14.12\end{array}$ \\
\hline $\begin{array}{l}\text { E. benzoate + Pyreth. } \\
(1: 2)\end{array}$ & $\begin{array}{l}\text { Conc. } \\
\mathrm{C} \%\end{array}$ & $\begin{array}{c}2.007 \pm 0.0041 \\
-30.60\end{array}$ & $\begin{array}{c}4.661 \pm 0.004 \mathrm{k} \\
-3.78\end{array}$ & $\begin{array}{c}3.742 \pm 0.005 \mathrm{f} \\
60.46\end{array}$ & $\begin{array}{c}2.689 \pm 0.004 \mathrm{f} \\
138.81\end{array}$ \\
\hline $\begin{array}{l}\text { Chlorflu. + Chlorpyr. } \\
(2: 1)\end{array}$ & $\begin{array}{l}\text { Conc. } \\
\mathrm{C} \%\end{array}$ & $\begin{array}{c}5.471 \pm 0.004 \mathrm{~d} \\
89.18\end{array}$ & $\begin{array}{c}2.632 \pm 0.005 p \\
-45.66\end{array}$ & $\begin{array}{c}3.016 \pm 0.004 \mathrm{~g} \\
29.33\end{array}$ & $\begin{array}{c}0.966 \pm 0.004 \mathrm{n} \\
-14.21\end{array}$ \\
\hline $\begin{array}{l}\text { Chlorflu. + E. benzoate } \\
(2: 1)\end{array}$ & $\begin{array}{l}\text { Conc. } \\
\mathrm{C} \%\end{array}$ & $\begin{array}{c}1.864 \pm 0.003 n \\
-35.55\end{array}$ & $\begin{array}{c}8.671 \pm 0.005 \mathrm{c} \\
79.00\end{array}$ & $\begin{array}{c}2.792 \pm 0.005 \mathrm{~h} \\
19.73\end{array}$ & $\begin{array}{c}3.443 \pm 0.004 \mathrm{c} \\
205.77\end{array}$ \\
\hline $\begin{array}{l}\text { Chlorflu. + Pyreth. } \\
(2: 1)\end{array}$ & $\begin{array}{l}\text { Conc. } \\
\text { C\% }\end{array}$ & $\begin{array}{c}4.918 \pm 0.003 \mathrm{f} \\
70.06\end{array}$ & $\begin{array}{l}3.703 \pm 0.0031 \\
-23.55\end{array}$ & $\begin{array}{c}0.921 \pm 0.004 p \\
-60.51\end{array}$ & $\begin{array}{c}2.121 \pm 0.004 \mathrm{i} \\
88.37\end{array}$ \\
\hline $\begin{array}{l}\text { Chlorpyr. + E. benzoate } \\
(2: 1)\end{array}$ & $\begin{array}{l}\text { Conc. } \\
\mathrm{C} \%\end{array}$ & $\begin{array}{c}0.422 \pm 0.003 q \\
-84.72\end{array}$ & $\begin{array}{c}2.345 \pm 0.004 \mathrm{r} \\
-51.59\end{array}$ & $\begin{array}{c}2.600 \pm 0.040 \mathrm{i} \\
11.49\end{array}$ & $\begin{array}{c}1.268 \pm 0.0031 \\
12.61\end{array}$ \\
\hline $\begin{array}{l}\text { Chlorpyr. + Pyreth. } \\
(2: 1)\end{array}$ & $\begin{array}{l}\text { Conc. } \\
\mathrm{C} \%\end{array}$ & $\begin{array}{c}3.766 \pm 0.004 \mathrm{~h} \\
30.22\end{array}$ & $\begin{array}{c}5.193 \pm 0.004 \mathrm{~h} \\
7.20\end{array}$ & $\begin{array}{c}4.116 \pm 0.004 \mathrm{e} \\
76.50\end{array}$ & $\begin{array}{c}1.406 \pm 0.004 \mathrm{k} \\
24.87\end{array}$ \\
\hline $\begin{array}{l}\text { E. benzoate + Pyreth. } \\
(2: 1)\end{array}$ & $\begin{array}{c}\text { Conc. } \\
\mathrm{C} \% \\
\end{array}$ & $\begin{array}{c}4.923 \pm 0.004 \mathrm{f} \\
70.23 \\
\end{array}$ & $\begin{array}{c}8.238 \pm 0.002 \mathrm{~d} \\
70.07 \\
\end{array}$ & $\begin{array}{c}2.778 \pm 0.004 \mathrm{~h} \\
19.13 \\
\end{array}$ & $\begin{array}{c}0.882 \pm 0.004 \mathrm{o} \\
-21.67 \\
\end{array}$ \\
\hline
\end{tabular}

- Values given are mean of three analysis.

- Means with the same letter in each column are not significant different $(P<0.05)$.

* Conc. $=$ concentration $* * \mathrm{C} \%=$ change percentage

$* * *$ Means $=$ mg protein $/ \mathrm{g}$ body weight $* * * * \mathrm{mg}$ lipid/ $\mathrm{g}$ body weight

\section{The total lipids:}

Data in Table (3) show that the mixtures of the tested compounds significantly increased the level of the total lipids throughout the two experimental periods comparing with the control. Obvious increase in the lipid levels took place in case of mixtures chlorfluazuron: chlorpyrifos (1:1) and chlorfluazuron: pyrethrins (1:2) indicating 190.91 and $316.96 \%$ after one and three days of treatment, respectively. Mead (2006) reported that the total lipid level in S. littoralis was reduced by treatment with different types of pesticides.

\section{Carbohydrate hydrolyzing enzymes:}

Results obtained in Table (4) cleared the changes in invertase, trehalase and amylase activity.

\section{Invertase.}

Significant reduction in invertase activity was shown after 1 day of treatment. The highest reduction percentage was found in case of chlorfluazuron: chlorpyrifos at level $2: 1$ recording $-88.68 \%$. The picture 
differed after 3 days, i.e., the treatments were, however, significantly increased the invertase activity and the highest increase percentage took place in case of the mixture chlorfluazuron: emamectin benzoate at level 1:2 indicating $260.78 \%$.

Table 4. Changes in carbohydrases enzymes activities in $4^{\text {th }}$ larval instars of $S$. littoralis treated with the tested compound's mixture.

\begin{tabular}{|c|c|c|c|c|c|c|c|}
\hline \multirow{2}{*}{ Treatment } & & \multicolumn{2}{|c|}{ Invertase $* * * *$} & \multicolumn{2}{|c|}{ Trehalase $* * * *$} & \multicolumn{2}{|c|}{ Amylase $* * * *$} \\
\hline & & 1 day & 3 days & 1 day & 3 days & 1 day & 3 days \\
\hline Control & Conc.* & $0.053 \pm 0.004 \mathrm{e}$ & $0.051 \pm 0.004 \mathrm{hi}$ & $0.053 \pm 0.005 \mathrm{~d}$ & $0.070 \pm 0.004 \mathrm{bc}$ & $0.057 \pm 0.004 \mathrm{~cd}$ & $0.043 \pm 0.004 \mathrm{hi}$ \\
\hline Chlorflu. + chlorpyr. & $\mathrm{SA}^{* *}$ & $0.040 \pm 0.004 \mathrm{fg}$ & $0.058 \pm 0.004 \mathrm{gh}$ & $0.015 \pm 0.004 \mathrm{hij}$ & $0.049 \pm 0.004 \mathrm{efg}$ & $0.055 \pm 0.003 \mathrm{cde}$ & $0.059 \pm 0.004 \mathrm{def}$ \\
\hline$(1: 1)$ & $\mathrm{RA} \% * * *$ & -24.53 & 13.73 & -71.70 & -30.00 & -3.51 & 37.21 \\
\hline Chlorflu. + E. & SA & $0.029 \pm 0.004 \mathrm{hi}$ & $0.077 \pm 0.004 \mathrm{e}$ & $0.021 \pm 0.004 \mathrm{gh}$ & $0.033 \pm 0.004 \mathrm{i}$ & $0.054 \pm 0.004 \mathrm{de}$ & $0.016 \pm 0.0041$ \\
\hline benzoate $(1: 1)$ & RA $\%$ & -45.28 & 50.98 & -60.38 & -52.86 & -5.26 & -62.79 \\
\hline Chlorflu. + Pyreth. & SA & $0.013 \pm 0.003 \mathrm{jk}$ & $0.070 \pm 0.003 \mathrm{ef}$ & $0.047 \pm 0.003 \mathrm{de}$ & $0.002 \pm 0.001 \mathrm{j}$ & $0.064 \pm 0.004 b c$ & $0.024 \pm 0.003 \mathrm{kl}$ \\
\hline$(1: 1)$ & $\mathrm{RA} \%$ & -75.47 & 37.25 & -11.32 & -97.14 & 12.28 & -44.19 \\
\hline Chlorpyr. + E. & SA & $0.049 \pm 0.004 \mathrm{ef}$ & $0.061 \pm 0.004 \mathrm{fg}$ & $0.069 \pm 0.004 \mathrm{c}$ & $0.050 \pm 0.004 \mathrm{efg}$ & $0.014 \pm 0.004 \mathrm{j}$ & $0.054 \pm 0.004 \mathrm{efg}$ \\
\hline benzoate $(1: 1)$ & $\mathrm{RA} \%$ & -7.55 & 19.61 & 30.19 & -28.57 & -75.44 & 25.58 \\
\hline Chlorpyr. + Pyreth. & SA & $0.020 \pm 0.003 \mathrm{ij}$ & $0.078 \pm 0.003 \mathrm{e}$ & $0.049 \pm 0.002 \mathrm{de}$ & $0.041 \pm 0.004 \mathrm{ghi}$ & $0.020 \pm 0.002 \mathrm{ij}$ & $0.059 \pm 0.002 \mathrm{def}$ \\
\hline$(1: 1)$ & $\mathrm{RA} \%$ & -62.26 & 52.94 & -7.55 & -41.43 & -64.91 & 37.21 \\
\hline E. benzoate + Pyreth. & SA & $0.054 \pm 0.003 \mathrm{e}$ & $0.071 \pm 0.004 \mathrm{e}$ & $0.008 \pm 0.002 \mathrm{ijk}$ & $0.063 \pm 0.002 \mathrm{~cd}$ & $0.016 \pm 0.003 \mathrm{ij}$ & $0.071 \pm 0.003 c$ \\
\hline$(1: 1)$ & RA $\%$ & 1.89 & 39.22 & -84.91 & -10.00 & -71.93 & 65.12 \\
\hline Chlorflu. + Chlorpyr. & SA & $0.042 \pm 0.004 \mathrm{fg}$ & $0.051 \pm 0.005 \mathrm{hi}$ & $0.016 \pm 0.004 \mathrm{hi}$ & $0.050 \pm 0.005 \mathrm{efg}$ & $0.089 \pm 0.004 \mathrm{a}$ & $0.044 \pm 0.004 \mathrm{ghi}$ \\
\hline$(1: 2)$ & $\mathrm{RA} \%$ & -20.75 & 0.00 & -69.81 & -28.57 & 56.14 & 2.33 \\
\hline Chlorflu. + E. benzoate & $\mathrm{SA}$ & $0.117 \pm 0.004 b$ & $0.184 \pm 0.004 \mathrm{a}$ & $0.040 \pm 0.005 \mathrm{ef}$ & $0.079 \pm 0.003 b$ & $0.024 \pm 0.003 \mathrm{hi}$ & $0.063 \pm 0.004 \mathrm{cde}$ \\
\hline$(1: 2)$ & $\mathrm{RA} \%$ & 120.75 & 260.78 & -24.53 & 12.86 & -57.89 & 46.51 \\
\hline Chlorflu. + Pyreth. & SA & $0.039 \pm 0.002 \mathrm{~g}$ & $0.014 \pm 0.003 \mathrm{k}$ & $0.005 \pm 0.002 \mathrm{jk}$ & $0.047 \pm 0.002 \mathrm{fgh}$ & $0.049 \pm 0.002 \mathrm{def}$ & $0.171 \pm 0.002 \mathrm{a}$ \\
\hline$(1: 2)$ & RA $\%$ & -26.42 & -72.55 & -90.57 & -32.86 & -14.04 & \\
\hline Chlorpyr. + E. & SA & $0.166 \pm 0.004 \mathrm{a}$ & $0.127 \pm 0.003 c$ & $0.040 \pm 0.004 \mathrm{ef}$ & $0.110 \pm 0.004 \mathrm{a}$ & $0.068 \pm 0.002 b$ & $0.051 \pm 0.003 \mathrm{fgh}$ \\
\hline te $(1: 2)$ & $\mathrm{RA} \%$ & 213.21 & 149.02 & -24.53 & 57.14 & 19.30 & 18.60 \\
\hline Chlorpyr. + Pyreth. & SA & $0.039 \pm 0.002 \mathrm{~g}$ & $0.048 \pm 0.002 \mathrm{i}$ & $0.083 \pm 0.002 b$ & $0.037 \pm 0.002 \mathrm{hi}$ & $0.024 \pm 0.002 \mathrm{hi}$ & $0.065 \pm 0.002 \mathrm{~cd}$ \\
\hline$(1: 2)$ & $\mathrm{RA} \%$ & -26.42 & -5.88 & 56.60 & -47.14 & -57.89 & 51.16 \\
\hline E. benzoate + Pyreth. & SA & $0.119 \pm 0.003 b$ & $0.046 \pm 0.003 \mathrm{ij}$ & $0.031 \pm 0.003 \mathrm{fg}$ & $0.036 \pm 0.003 \mathrm{i}$ & $0.055 \pm 0.002 \mathrm{cde}$ & $0.046 \pm 0.003 \mathrm{ghi}$ \\
\hline$(1: 2)$ & RA $\%$ & 124.53 & -9.80 & -41.51 & -48.57 & -3.51 & 6.98 \\
\hline Chlorflu. + & SA & $0.006 \pm 0.004 \mathrm{k}$ & $0.108 \pm 0.003 \mathrm{~d}$ & $0.069 \pm 0.003 \mathrm{c}$ & $0.054 \pm 0.004 \mathrm{def}$ & $0.032 \pm 0.004 \mathrm{gh}$ & $0.058 \pm 0.004 \mathrm{def}$ \\
\hline Chlorpyr.(2:1) & RA\% & -88.68 & 111.76 & 30.19 & & -43.86 & 34.88 \\
\hline Chlorflu. + E. & SA & $0.077 \pm 0.003 \mathrm{c}$ & $0.076 \pm 0.003 \mathrm{e}$ & $0.004 \pm 0.004 \mathrm{k}$ & $0.059 \pm 0.004 \mathrm{de}$ & $0.040 \pm 0.005 \mathrm{fg}$ & $0.050 \pm 0.005 \mathrm{fgh}$ \\
\hline benzoate $(2: 1)$ & RA\% & 45.28 & 49.02 & -92.45 & & -29.82 & 16.28 \\
\hline Chlorflu. + Pyreth. & SA & $0.016 \pm 0.003 \mathrm{j}$ & $0.045 \pm 0.003 \mathrm{ij}$ & $0.094 \pm 0.003 \mathrm{a}$ & $0.032 \pm 0.003 \mathrm{i}$ & $0.089 \pm 0.002 \mathrm{a}$ & $0.031 \pm 0.002 \mathrm{jk}$ \\
\hline$(2: 1)$ & $\mathrm{RA} \%$ & -69.81 & -11.76 & 77.36 & -54.29 & 56.14 & -27.91 \\
\hline Chlorpyr. + E. & SA & $0.016 \pm 0.004 \mathrm{j}$ & $0.147 \pm 0.003 b$ & $0.091 \pm 0.003 \mathrm{ab}$ & $0.050 \pm 0.00 \mathrm{efg}$ & $0.011 \pm 0.004 \mathrm{j}$ & $0.085 \pm 0.004 \mathrm{~b}$ \\
\hline benzoate $(2: 1)$ & RA\% & -69.81 & 188.24 & 71.70 & -28.57 & -80.70 & 97.67 \\
\hline Chlorpyr. + Pyreth. & SA & $0.064 \pm 0.003 \mathrm{~d}$ & $0.053 \pm 0.003 \mathrm{ghi}$ & $0.028 \pm 0.002 \mathrm{~g}$ & $0.032 \pm 0.003 \mathrm{i}$ & $0.072 \pm 0.002 b$ & $0.036 \pm 0.002 \mathrm{ij}$ \\
\hline$(2: 1)$ & $\mathrm{RA} \%$ & 20.75 & 3.92 & -47.17 & -54.29 & 26.32 & -16.28 \\
\hline E. benzoate + & SA & $0.036 \pm 0.003 \mathrm{gh}$ & $0.037 \pm 0.003 \mathrm{j}$ & $0.021 \pm 0.004 \mathrm{gh}$ & $0.074 \pm 0.003 b$ & $0.047 \pm 0.002 \mathrm{ef}$ & $0.059 \pm 0.003 \mathrm{def}$ \\
\hline Pyreth. (2:1) & RA $\%$ & -32.08 & -27.45 & -60.38 & 5.71 & -17.54 & 37.21 \\
\hline
\end{tabular}

- Values given are mean of three analysis.

- Means with the same letter in each column are not significant different $(\mathbf{P}<0.05)$.

* Conc. $=$ concentration $* * \mathbf{S A}=$ specific activity $* * * \mathbf{R A} \%=$ relative activity percentage

$* * * *$ Means $=\mu \mathrm{g}$ glucose $/ \mathrm{g}$ body weight

\section{Trehalase.}

Most of treatments recorded reduction effects on trehalase activity after 1 and 3 days of treatment compared with the control. The reduction percentages ranged between -7.55 to $-97.14 \%$ during the times of the experiments.

Amylase.

Smilar to invertase and trehalase, significant reduction in amylase activity was noticed after 1 day of treatment. The highest reduction percentage $(-80.70 \%)$ was observed in mixture of chlorpyrifos: emamectin benzoate at level 2:1. In contrast, after 3 days, the tested mixtures were, however, increased the amylase activity to $297.67 \%$ in case of chlorfluazuron: pyrethrins at level 1:2.

Carbohydrates are the one of main importance source of energy or conversion to lipids or proteins. Carbohydrate hydrolyzing enzymes controlling the carbohydrates metabolism, the increase of these enzymes during the larval stage degrade carbohydrates to glucose for chitin build-up
(Wyatt, 1967), The disturbance of trehalase activity might hamper the supply of glucose needed for chitin build up (Kandy and Killy, 1962; Shakoori et al., 1998; Kheder, 2002 and Younes et al., 2008).

Threfore, the inhibition of carbohydrate hydrolyzing enzymes might affect the molting process and subsequently may explained the reason of mortality occurred in larvae as illustrated previously in the toxicological experiments. These results agree with previous research who observed marked reduction in the carbohydrate hydrolyzing enzymes specifically amylase and invertase was observed aftr treated $5^{\text {th }}$ instar larvae of cotton leafworm, $S$. littoralis (Lepidoptera: Noctuidae) with sub-lethal concentrations of thuringeinsin (beta-exotoxin of B. thuringiensis). (IGRs) in $S$. littoralis larvae treated with insecticdes were generally decreased than untreated larvae during diffrent tested times ( El-Ghar et al., 1995; Eid, 2002; Mead et al., 2008 and Al-shannaf et al., 2012) 


\section{Transaminase enzymes.}

On ground of the activities of AST enzymes (Table 5), it is clear that the highest significant increase of the enzyme activity was observed with the mixtures of chlorfluazuron: chlorpyrifos at level $1: 1$ and chlorfluazuron: pyrethrins (1:2) after one and three days' post treatment, respectively. The respective increase percentages were 113.19 and 125.13 . Concerning the activity of ALT enzyme, obvious increase percentages were 118.93 and 245.28 after one and three days of the experiment with the mixtures of chlorpyrifos: pyrethrins at level 2:1 and chlorfluazuron: pyrethrins (1:2), respectively.

Table 5. Changes in transaminase enzymes activities in $4^{\text {th }}$ larval instars $S$. littoralis treated with the tested compound's mixture.

\begin{tabular}{|c|c|c|c|c|c|}
\hline \multirow{2}{*}{ Treatment } & & \multicolumn{2}{|c|}{ AST $* * * *$} & \multicolumn{2}{|c|}{ ALT**** } \\
\hline & & 1 days & 3 days & 1 days & 3 days \\
\hline$\overline{\text { Control }}$ & Conc. $^{*}$ & $1.190 \pm 0.003 \mathrm{j}$ & $0.589 \pm 0.004 \mathrm{~m}$ & $17.786 \pm 0.002 \mathrm{n}$ & $9.821 \pm 0.002 \mathrm{~s}$ \\
\hline \multirow{2}{*}{ Chlorflu. + Chlorpyr.(1:1) } & $\mathrm{SA}^{* *}$ & $2.537 \pm 0.004 \mathrm{a}$ & $0.881 \pm 0.003 \mathrm{~g}$ & $35.915 \pm 0.003 b$ & $14.875 \pm 0.002 \mathrm{k}$ \\
\hline & $\mathrm{RA} \% * * *$ & 113.19 & 49.58 & 101.93 & 51.46 \\
\hline \multirow{2}{*}{ Chlorflu. + E. benzoate $(1: 1)$} & SA & $1.066 \pm 0.003 \mathrm{~m}$ & $0.662 \pm 0.004 \mathrm{k}$ & $12.248 \pm 0.003 \mathrm{~s}$ & $16.280 \pm 0.003 \mathrm{i}$ \\
\hline & RA\% & -10.42 & 12.39 & -31.14 & 65.77 \\
\hline \multirow{2}{*}{ Chlorflu. + Pyreth. (1:1) } & SA & $1.120 \pm 0.0031$ & $0.982 \pm 0.004 \mathrm{e}$ & $21.004 \pm 0.0021$ & $19.803 \pm 0.002 f$ \\
\hline & $\mathrm{RA} \%$ & -5.88 & 66.72 & 18.09 & 101.64 \\
\hline \multirow{2}{*}{ Chlorpyr. + E. benzoate $(1: 1)$} & SA & $1.355 \pm 0.002 \mathrm{f}$ & $0.369 \pm 0.003 p$ & $23.168 \pm 0.002 \mathrm{i}$ & $11.531 \pm 0.003 \mathrm{o}$ \\
\hline & $\mathrm{RA} \%$ & 13.87 & -37.35 & 30.26 & 17.41 \\
\hline \multirow{2}{*}{ Chlorpyr. + Pyreth.(1:1) } & SA & $1.313 \pm 0.003 \mathrm{~g}$ & $0.973 \pm 0.003 \mathrm{e}$ & $19.016 \pm 0.002 \mathrm{~m}$ & $14.792 \pm 0.0031$ \\
\hline & RA\% & 10.34 & 65.20 & 6.92 & 50.62 \\
\hline \multirow{2}{*}{ E. benzoate + Pyreth.(1:1) } & SA & $1.605 \pm 0.003 \mathrm{~d}$ & $0.722 \pm 0.004 \mathrm{i}$ & $17.387 \pm 0.003 \mathrm{o}$ & $10.831 \pm 0.003 q$ \\
\hline & $\mathrm{RA} \%$ & 34.87 & 22.58 & -2.24 & 10.28 \\
\hline \multirow{2}{*}{ Chlorflu. + Chlorpyr.(1:2) } & SA & $1.675 \pm 0.003 b$ & $0.512 \pm 0.003 \mathrm{o}$ & $27.032 \pm 0.003 \mathrm{f}$ & $10.486 \pm 0.002 \mathrm{r}$ \\
\hline & RA\% & 40.76 & -13.07 & 51.98 & 6.77 \\
\hline \multirow{2}{*}{ Chlorflu.+ E. benzoate $(1: 2)$} & SA & $1.310 \pm 0.003 \mathrm{~g}$ & $1.093 \pm 0.003 b$ & $17.114 \pm 0.003 p$ & $22.654 \pm 0.003 \mathrm{e}$ \\
\hline & RA\% & 10.08 & 85.57 & -3.78 & 130.67 \\
\hline \multirow{2}{*}{ Chlorflu. + Pyreth.(1:2) } & SA & $1.622 \pm 0.003 \mathrm{c}$ & $1.326 \pm 0.003 \mathrm{a}$ & $23.275 \pm 0.003 \mathrm{~h}$ & $33.910 \pm 0.002 \mathrm{a}$ \\
\hline & RA\% & 36.30 & 125.13 & 30.86 & 245.28 \\
\hline \multirow{2}{*}{ Chlorpyr. + E. benzoate $(1: 2)$} & SA & $1.196 \pm 0.002 \mathrm{j}$ & $0.556 \pm 0.002 \mathrm{n}$ & $24.695 \pm 0.003 \mathrm{~g}$ & $10.750 \pm 0.003 p$ \\
\hline & RA\% & 0.50 & -5.60 & 38.85 & 9.46 \\
\hline \multirow{2}{*}{ Chlorpyr. + Pyreth.(1:2) } & SA & $0.950 \pm 0.003 \mathrm{o}$ & $0.789 \pm 0.002 \mathrm{~h}$ & $30.660 \pm 0.004 d$ & $25.440 \pm 0.005 \mathrm{c}$ \\
\hline & $\mathrm{RA} \%$ & -20.17 & 33.96 & 72.38 & 159.04 \\
\hline \multirow{2}{*}{ E. benzoate + Pyreth.(1:2) } & SA & $1.457 \pm 0.013 \mathrm{e}$ & $0.605 \pm 0.0041$ & $32.715 \pm 0.004 \mathrm{c}$ & $24.124 \pm 0.003 \mathrm{~d}$ \\
\hline & RA\% & 22.44 & 2.72 & 83.94 & 145.64 \\
\hline \multirow{2}{*}{ Chlorflu. + Chlorpyr.(2:1) } & SA & $0.934 \pm 0.002 p$ & $0.591 \pm 0.003 \mathrm{~m}$ & $21.696 \pm 0.003 \mathrm{j}$ & $13.714 \pm 0.003 \mathrm{~m}$ \\
\hline & RA\% & -21.51 & 0.34 & 21.98 & 39.64 \\
\hline \multirow{2}{*}{ Chlorflu. + E. benzoate $(2: 1)$} & SA & $1.275 \pm 0.002 \mathrm{~h}$ & $1.039 \pm 0.003 \mathrm{c}$ & $12.922 \pm 0.003 q$ & $30.738 \pm 0.003 b$ \\
\hline & RA\% & 7.14 & 76.40 & -27.35 & 212.98 \\
\hline Chlorflu. + Pyreth.(2:1) & SA & $0.871 \pm 0.003 q$ & $0.934 \pm 0.003 \mathrm{f}$ & $12.454 \pm 0.002 \mathrm{r}$ & $16.482 \pm 0.003 \mathrm{~h}$ \\
\hline \multirow{2}{*}{ Chlorpyr.+E. benzoate $(2: 1)$} & RA\% & -26.81 & 58.57 & -29.98 & 67.82 \\
\hline & SA & $1.259 \pm 0.002 \mathrm{i}$ & $0.677 \pm 0.003 \mathrm{j}$ & $27.540 \pm 0.002 \mathrm{e}$ & $17.007 \pm 0.003 \mathrm{~g}$ \\
\hline \multirow{2}{*}{ Chlorpyr. + Pyreth.(2:1) } & RA\% & 5.80 & 14 & 54.84 & 73.17 \\
\hline & $\begin{array}{l}\text { SA } \\
\text { DA }\end{array}$ & $1.041 \pm 0.004 n$ & $0.999 \pm 0.004 d$ & $38.939 \pm 0.002 \mathrm{a}$ & $15.301 \pm 0.002 \mathrm{~J}$ \\
\hline \multirow{3}{*}{ E. benzoate + Pyreth.(2:1) } & RA\% & -12.52 & 69.61 & 118.93 & 55.80 \\
\hline & SA & $1.145 \pm 0.003 \mathrm{k}$ & $0.305 \pm 0.003 q$ & $21.184 \pm 0.004 \mathrm{k}$ & $12.669 \pm 0.002 \mathrm{n}$ \\
\hline & RA\% & 5.10 & & 19.10 & \\
\hline
\end{tabular}

- Values given are mean of three analysis.

- Means with the same letter in each column are not significant different $(P<0.05)$.

* Conc. $=$ concentration $*$ SA= specific activity

$* * * \mathbf{R A} \%=$ relative activity percentage

$* * * *$ Means $=\mu$ g pyruvate $/ g$ body weight

ALT acts as a catalytic agent in carbohydrates metabolism and also the key enzymes in the formation of non-essential amino acids, in metabolism of nitrogen waste, gluconeogenesis and correlated with protein anabolism and catabolism (Katumuma, et al. 1968; Mordue and Goldsworthy, 1973).

The increasing ALT and AST activity in haemolymph of larvae, to some extent, in agreement with the reported for several IGRs or insecticides, such as pyriproxyfen, flufenoxuron or teflubenzuron (ElKordy et al., 1995), hexaflumuron alone or its binary mixture with chlorpyriphos (Mohamed and Azab, 2002; Zohry, 2006). The increasing activity of transaminases may be due to the occurrence of reversible binding between the tested compounds and enzymatic site of action on the enzyme surface. Relationships between protein synthesis and transaminase levels were affected by the hormonal control of protein synthesis and neurosecretory hormones which involved in the regulation of transaminase levels (Tanani et al., 2016)

\section{Acid and alkaline phosphatase.}

Data in Table (6) indicated that, the activities of acid phosphatase enzyme was generally reduced at different levels as affected by all treatments than control after 1 day of treatment, but after 3 days of treatment most of insecticides' combination caused an increase of the enzyme at different levels than the orginal level, where chlorfluazuron: emamectin benzoate at level 1:1 gave the highest significant 
increase of ACP recording $43.897 \pm 0.002 \mu \mathrm{g}$ phenol/ $\mathrm{g}$ body weight $(194.97 \%)$. The same table cleared that the tested mixtures reduced the levels of ALP enzyme's activity after 1 and 3 days. The highest significant reduction percentages were recorded with the mixtures of chlorpyrifos: pyrethrins (2:1) and chlorfluazuron: emamectin benzoate (2:1) indicating $\quad-77.45$ and $-82.60 \%$ after one and three days of the experiment, respectively.
Also, the inhibition in the activity of both acid and alkaline phosphatases was obtained by Eid (2002) who found great reduction in the activities of them for all tested strains of $S$. littoralis using chlorpyrifos. In continuity, Abd El-Mageed et al., (2008) mentioned that the change in response to tested biocides in S. littoralis larvae could be associated with the decrease in alkaline phosphatase activity and varied effect in acid phosphates activity.

Table 6 . Changes in acid phosphatase $\&$ alkaline phosphatase enzymes activities in $4^{\text {th }}$ larval instars of $S$. littoralis treated with the tested compound's mixture.

\begin{tabular}{|c|c|c|c|c|c|}
\hline \multirow{2}{*}{ Treatment } & & \multicolumn{2}{|c|}{$\mathrm{ACP} * * * *$} & \multicolumn{2}{|c|}{$\mathbf{A L P} * * * *$} \\
\hline & & 1 day & 3 days & 1 day & 3 days \\
\hline Control & Conc.* & $23.109 \pm 0.004 \mathrm{a}$ & $14.882 \pm 0.004 j$ & $23.473 \pm 0.003 \mathrm{f}$ & $37.706 \pm 0.003 b$ \\
\hline \multirow{2}{*}{ Chlorflu. + Chlorpyr.(1:1) } & $\mathrm{SA}^{* *}$ & $8.668 \pm 0.003 \mathrm{o}$ & $17.298 \pm 0.003 \mathrm{i}$ & $6.328 \pm 0.002 \mathrm{r}$ & $27.730 \pm 0.004 \mathrm{e}$ \\
\hline & $\mathrm{RA} \% * * *$ & -62.49 & 16.23 & -73.04 & -26.46 \\
\hline \multirow{2}{*}{ Chlorflu. + E. benzoate(1:1) } & SA & $15.955 \pm 0.004 \mathrm{c}$ & $43.897 \pm 0.002 \mathrm{a}$ & $22.207 \pm 0.003 \mathrm{~g}$ & $7.594 \pm 0.004 \mathrm{r}$ \\
\hline & RA\% & -30.96 & 194.97 & -5.39 & -79.86 \\
\hline \multirow{2}{*}{ Chlorflu. + Pyreth.(1:1) } & SA & $9.358 \pm 0.0021$ & $7.211 \pm 0.003 \mathrm{r}$ & $28.037 \pm 0.003 \mathrm{~d}$ & $11.928 \pm 0.003 p$ \\
\hline & $\mathrm{RA} \%$ & -59.50 & -51.55 & 19.44 & -68.37 \\
\hline \multirow{2}{*}{ Chlorpyr. + E. benzoate(1:1) } & $\mathrm{SA}$ & $16.071 \pm 0.003 \mathrm{~b}$ & $28.536 \pm 0.003 \mathrm{~d}$ & $25.199 \pm 0.002 \mathrm{e}$ & $18.372 \pm 0.0041$ \\
\hline & $\mathrm{RA} \%$ & -30.46 & 91.75 & 7.35 & -51.28 \\
\hline \multirow{2}{*}{ Chlorpyr. + Pyreth.(1:1) } & $\mathrm{SA}$ & $11.084 \pm 0.003 \mathrm{i}$ & $21.210 \pm 0.003 \mathrm{~g}$ & $9.512 \pm 0.002 p$ & $22.514 \pm 0.003 \mathrm{j}$ \\
\hline & $\mathrm{RA} \%$ & -52.04 & 42.52 & -59.48 & -40.29 \\
\hline \multirow{2}{*}{ E. benzoate + Pyreth.(1:1) } & SA & $11.621 \pm 0.004 \mathrm{~h}$ & $14.153 \pm 0.003 \mathrm{k}$ & $17.375 \pm 0.004 \mathrm{i}$ & $17.451 \pm 0.003 \mathrm{~m}$ \\
\hline & $\mathrm{RA} \%$ & -49.71 & -4.90 & -25.98 & -53.72 \\
\hline \multirow{2}{*}{ Chlorflu. + Chlorpyr.(1:2) } & SA & $8.285 \pm 0.003 p$ & $33.023 \pm 0.003 b$ & $46.140 \pm 0.003 \mathrm{a}$ & $74.906 \pm 0.003 \mathrm{a}$ \\
\hline & $\mathrm{RA} \%$ & -64.15 & 121.90 & 96.57 & 98.66 \\
\hline \multirow{2}{*}{ Chlorflu. + E. benzoate(1:2) } & SA & $9.320 \pm 0.004 \mathrm{~m}$ & $11.161 \pm 0.004 \mathrm{n}$ & $18.985 \pm 0.002 \mathrm{~h}$ & $33.483 \pm 0.003 d$ \\
\hline & $\mathrm{RA} \%$ & -59.67 & -25.00 & -19.12 & -11.20 \\
\hline \multirow{2}{*}{ Chlorflu. + Pyreth.(1:2) } & SA & $9.090 \pm 0.006 \mathrm{n}$ & $11.276 \pm 0.003 \mathrm{~m}$ & $9.895 \pm 0.003 n$ & $13.462 \pm 0.004 \mathrm{n}$ \\
\hline & $\mathrm{RA} \%$ & -60.66 & -24.23 & -57.85 & -64.30 \\
\hline \multirow{2}{*}{ Chlorpyr. + E. benzoate(1:2) } & $\mathrm{SA}$ & $9.934 \pm 0.004 \mathrm{k}$ & $26.081 \pm 0.003 \mathrm{f}$ & $9.857 \pm 0.004 \mathrm{o}$ & $18.755 \pm 0.003 \mathrm{k}$ \\
\hline & $\mathrm{RA} \%$ & -57.01 & 75.25 & -59.16 & -50.26 \\
\hline \multirow{2}{*}{ Chlorpyr. + Pyreth.(1:2) } & SA & $12.657 \pm 0.003 \mathrm{~g}$ & $8.285 \pm 0.003 q$ & $13.961 \pm 0.004 \mathrm{~m}$ & $26.043 \pm 0.003 f$ \\
\hline & $\mathrm{RA} \%$ & -45.23 & -44.33 & -40.52 & -30.93 \\
\hline \multirow{2}{*}{ E. benzoate + Pyreth.(1:2) } & SA & $6.635 \pm 0.003 q$ & $19.522 \pm 0.004 \mathrm{~h}$ & $15.457 \pm 0.003 \mathrm{k}$ & $23.741 \pm 0.003 \mathrm{~h}$ \\
\hline & $\mathrm{RA} \%$ & -71.29 & 31.18 & -34.15 & -37.04 \\
\hline \multirow{2}{*}{ Chlorflu. + Chlorpyr.(2:1) } & SA & $10.317 \pm 0.003 \mathrm{j}$ & $10.701 \pm 0.003 \mathrm{o}$ & $37.894 \pm 0.003 b$ & $25.813 \pm 0.003 \mathrm{~g}$ \\
\hline & $\mathrm{RA} \%$ & -55.36 & -28.09 & 61.44 & -31.54 \\
\hline \multirow{2}{*}{ Chlorflu. + E. benzoate(2:1) } & SA & $15.879 \pm 0.004 \mathrm{~d}$ & $26.963 \pm 0.003 \mathrm{e}$ & $16.684 \pm 0.0036 \mathrm{j}$ & $6.559 \pm 0.004 \mathrm{~s}$ \\
\hline & $\mathrm{RA} \%$ & -31.29 & 81.18 & -28.92 & -82.60 \\
\hline \multirow{2}{*}{ Chlorflu. + Pyreth.(2:1) } & SA & $14.882 \pm 0.003 \mathrm{e}$ & $11.660 \pm 0.0031$ & $15.227 \pm 0.0031$ & $7.748 \pm 0.003 q$ \\
\hline & RA\% & -35.60 & -21.65 & -35.13 & -79.45 \\
\hline \multirow{2}{*}{ Chlorpyr. + E. benzoate(2:1) } & SA & $3.260 \pm 0.004 \mathrm{~s}$ & $9.934 \pm 0.003 p$ & $6.367 \pm 0.003 q$ & $33.982 \pm 0.003 c$ \\
\hline & $\mathrm{RA} \%$ & -85.89 & -33.25 & -72.88 & -9.88 \\
\hline \multirow{2}{*}{ Chlorpyr. + Pyreth.(2:1) } & SA & $3.452 \pm 0.003 \mathrm{r}$ & $4.756 \pm 0.002 \mathrm{~s}$ & $5.293 \pm 0.003 \mathrm{~s}$ & $12.734 \pm 0.004 \mathrm{o}$ \\
\hline & RA\% & -85.06 & -68.04 & -77.45 & -66.23 \\
\hline \multirow{2}{*}{ E. benzoate + Pyreth. $(2: 1)$} & SA & $14.460 \pm 0.003 \mathrm{f}$ & $28.651 \pm 0.005 \mathrm{c}$ & $32.256 \pm 0.004 c$ & $22.706 \pm 0.003 \mathrm{i}$ \\
\hline & $\mathrm{RA} \%$ & -37.43 & 92.52 & 37.42 & -39.78 \\
\hline
\end{tabular}

- Values given are mean of three analysis.

- Means with the same letter in each column are not significant different $(P<0.05)$.

* Conc. $=$ concentration $* * \mathbf{S A}=$ specific activity

$* * * \mathbf{R A} \%=$ relative activity percentage

$* * * *$ Means $=\mu \mathrm{g}$ phenol $/ \mathrm{g}$ body weight

The phenomenon of potentiation presented in this set of experiments could be elucidated to the inhibition of detoxification mechanisms by one of the two toxicants in the mixture and thus spanning high titer of the other component to react with the specific target. Each behavior depends greatly on the permeability of each toxicant via the insect integument as well as the second barrier surrounding the target (e.g. nerve sheath) which is in turn depends greatly on the polarity, stability (rate of degradation), partitioning and storage of the test compounds. Indeed, this explanation could be noticed with compounds which have similar mode of action, with those having independent mode of action.
One expectation that potentiation is due to the level of affinity with the most vital insect component (i.e. enzymes) which in turn depend on the amount of each toxicants in the mixture.

\section{REFERENCES}

Abd El-Mageed, A. E. M. A.; Anwar, E. M. and Elgohary, L. R. A. (2008). Biochemical side effects of some commercial biocides on cotton leafworm. Arch. Phytopath. Plant Protec., 41(3): 227-232.

Abd El-Mageed, A. E. M. and Shalaby S. E. M. (2011). Toxicity and biochemical impacts of some new insecticide mixtures on cotton leafworm Spodoptera littoralis (Boisd.). Plant Protect. Sci., 47: 166-175. 
Abd El-Megeed, M. I.; Abbas, M. G.; Gadallah, A. I. and Hanafy, A. (2000). Resistance development and change of response of the cotton leafworm Spodoptera littoralis (Boisd.) to chitin synthesis inhibitors and their binary mixtures. Ann. Agric. Sci. (Cairo), 4: 1573-1583.

Ahmad, M. (2009). Observed potentiation between pyrethroid and organophosphate insecticides for the management of Spodoptera litura (Lepidoptera: Noctuidae). CropProt. 28: 264-268.

Al-shannaf, H. M.; Mead H. M. and Al-Kazafy, H. S. (2012). Toxic and Biochemical Effects of Some Bioinsecticides and IGRs on American Bollworm, Helicoverpa armigera (hüb.) (Noctuidae: lepidoptera) in Cotton Fields. J. Biofertil. Biopestici., 3:118.

Ascher, K. R. S.; Eliyahu, M.; Ishaaya, I.; Zur, M. and Ben-Moshe, E. (1986). Synergism of pyrethroidorganophosphorus insecticide mixtures in insects and their toxicity against Spodoptera littoralis larvae. Phytoparasitica, 14: 101-110.

Costat Statistical Software (2005). Microcomputer Program Analysis, Version 6. 311. CoHort Software, Mont., Calif.

Eid, A. M. (2002). Esterases and phosphatases in relation to chlorpyrifos resistance in Spodoptera littoralis (Boisd.) (Lepidoptera: Noctuidae). Egypt. J. Appl. Sci., 17: 275-284.

El-Bermawy, Z. A.; El-Shiekh A. A.; Rashwan; M. H. and Radwan, H. S. A. (1992). Pyrethroids resistance in Spodoptera littoralis (Boisd.) (Lepidoptera: Noctuidae) in lower egypt. Bull. Ent. Soc. Egypt Econ. Ser., 19: 41-51.

El-Defrawi, M. E.; Tapozad, A.; Mansour, N. and Zeid, M. (1964). Toxicological studies on the Egyptian cotton leafworm Prodenia littura (L.). 1.Susceptibility of different larval instars of P. littura to insecticides. J. Econ. Entomol., (57):591-593.

El-Ghar, G. E.; Radwan, H. S.; El-Bermawy, Z. A. and Zidan, L. T. (1995). Inhibitory effect of thuringiensin and abamectin on digestive enzymes and non-specific esterases of Spodoptera littoralis (Boisd.) (Lepidoptera: Noctuidae) larvae. J. Appl. Entomol., 119:355359.

El-Guindy, M. A.; Issa, Y. H.; El-Malla, M. A. and Abdel Fattah, I. (1985). Insect growth regulators as suppressors to insecticide resistance in a fenvalerate resistant strain of Spodoptera littoralis (Boisd.). Bull. Ent. Soc. Egypt Econ. Ser., 14: 373-383.

El-Kordy, M. W.; Gadallah, A. I.; Abbas, M. G. and Mostafa, S. A. (1995). Effect of pyriproxyfen, flufenoxuron and teflubenzuron on some biochemical aspects of Spodoptera littoralis. AlAzhar J. Agric. Res., 21: 223-238.

El-Sheikh, E. S. A. (2015). Comparative toxicity and sublethal effects of emamectin benzoate, lufenuron and spinosad on Spodoptera littoralis (Boisd.) (Lepidoptera: Noctuidae). Crop Protection, 67: 228-234.
Ghoneim, Y. F.; Singab, M.; Abou-Yousef, H. M. and AbdEl-Hai, N. S. (2012). Efficacy of certain insecticides and their mixtures with the tested IGRs against a field strain of the cotton leafworm, Spodoptera littoralis (Boisd.) under laboratory conditions. Aust. J. Basic Appl. Sci., 6 (6): 300-304.

Gornall, J. G.; Bardwill, G. J. and David, M. M. (1949). Determination of serum protein by mean of biuret reaction. J. Bio. Chem., 117: 751-766.

Guilabault, G. G. (1970). Enzymatic methods of analysis. Pergamon Press, Oxford, 347.

Ishaaya, I. and Swiriski, E. (1976). Trehalase, invertase and amylase activities in the black scle, Saissetia oleae and their relation to host adabebility, J. Ins. Physiol., 16: 1025-1029.

Kandil, M. A., Said, H. K.; Abbas, M. E. and Mahdy, A. A. M. (2006). The effect of insect growth regulators and their binary mixtures on laboratory strain of $S$. littoralis (Lepidoptera: Noctuidae). Bull. Ent. Soc. Egypt, Econ., 32: 47-63.

Kandy, D. J. and Killy, B. A. (1962). Studies on chitin synthesis in the desert locust. J. Exp. Biolo., 39: 129-140.

Katumuma, N.; Okada, M.; Katsumua, T., Fujino, A. and Matsuzawa, T. (1968). Different metabolic rates of tranaminases isozymes. In: "Pyridoxal catalysis: Enzymes and Modelsystems" (Shell, E.E.; Braunstein, A.E.; Severin, E.S. and Torchin Sky, Y.M., eds.). Inter Science, New York.

Kheder, M. M. (2002). Effect of some plant extracts on the insect growth regulators applied to control cotton leafworm on honey bee Aphis mellifera L. MSc., Thesis, Fac., Agric. Zagazig Univ. Egypt pp.204

Khidr, A.A.; Abdeen, S. A. O.; Eissa, M. A.; Azab, A. M. H. and Hassan, A. I. (2003). Evaluation of some insecticides and their joint action against the cotton leafworm, Spodoptera littoralis (Boisd.) on lettuce vegetable plant, Lactuca sativa L. Ann. Agric. Sci., Moshtohor, 41(3): 1323-1330.

Korrat, E. E. E.; Abdelmonem, A. E.; Helalia, A. A. R. and Khalifa, H. M. S. (2012). Toxicological study of some conventional and nonconventional insecticides and their mixtures against cotton leafworm, Spodoptera littoralis (Boisd.) (Lepidoptera: Noectudae). Ann. Agric. Sci. (Cairo), 57(2): 199-206.

Mansour, N. A.; El-Defrawi, M. E.; Tappozado, A. and Zeid M. (1966). Toxicological studies on the Egyptian cotton leafworm, Prodenia litura. IV: Potentiation and antagonism of organophosphorus compounds and carbamate insecticides. J. Econ. Entomol., $59: 307-11$.

Mead, Hala, M. I. (2006). Studies on biochemical and biological activities of some larvicidal agents on cotton leafworm, Spodoptera littoralis (Boisduval) (Lepidoptera: Noctuidae). Ph. D. Thesis, Fac. Sci., Suez Canal Univ., 220 pp.

Mead, Hala, M. I., El-Sheakh A. A.; Soliman B. A., Desuky, W. M. and Abo-Ghalia, A. H. (2008). Biochemical effect of some compounds on carbohydrate hydrolyzing enzymes of cotton leafworm, Spodoptera littoralis (Boisd.), Egypt. J Agric Res., 86: 2169-2192. 
Metayi, Mervat, H. A., Ibrahiem, Moataza, A. M. and ElDeeb, Dalia, A. (2015). Toxicity and Some Biological Effects of Emamectin Benzoate, Novaluron and Diflubenzuron against Cotton Leafworm. Alexandria Sci. Exchange J., 36(4): 350357.

Mohamed, H. A. and Azab, A. M. (2002). Effect of insect growth regulators and binary mixtures on enzymes activity of Egyptian cotton leafworm, Spodoptera littoralis, (Boisd.) Larvae.Proceeding of $2^{\text {nd }}$ Int. Conf. Plant Prot. Res. Inst., Cairo, Egypt, 1: 617-622.

Mordue, W. and Goldsworthy, G. J. (1973). Transaminase levels and uric acid production inadult locusts. Insect Biochem., 3: 419-427.

Radwan, H. S. A., Nassar, M. E., El-Shikh, A. E., Abd El-Razik, M. A. A. (2009). Joint action of bioinsecticides and their role in development of resistance in Spodoptera littoralis (Boisd.). Minufiya. J. Agric. Res., 34 (2), 775-788.

Rashwan, M. H., El-Bermaway, Z. A.; El-Sheikh, A. E. and Radwan, H. S. A. (1992). The onset of organophosphates and carbamates resistance among Lower Egypt population of the cotton leafworm spodoptera littoralis (Boisd.). Bull. Ent. Soc. Egypt, Econ. Ser., 19: 211-220.

Reitman, S. M. and Frankel, S. (1957). A colorimetric method for determination of serum glutamic pyruvic transaminase. Am. J. Clin. Path., 28: 56-63.

Schmit (1964). Colorimetric determination of serum total lipids using sulfophosphovanilic mixture. $\mathrm{Ph}$. D Thesis, Lyon. Unvi., France.

Shakoori, A. R.; Salem, M. A. and Mantle, D. (1998). Some macromolecular abnormalities induced by a sub lethal dose of Cymbush $10 \mathrm{EC}$ in adult beetle of Tribolium castaneum. Pakistan. J. Zool., 30(2): 83-90

Shonouda, M. L.; Farrag, R. M. and Salama, O. M. (2000). Efficacy of the botanical extracts (myrrh), chemical insecticides and their combinations on the cotton leafworm, Spodoptera littoralis (Boisd.) (Lepidoptera: Noctuidae). J. Environ. Sci. Heal. B., 35(3): 347-356.
Sun, Y. P. (1950). Toxicity index. An improved method of comparing the relative toxicity of insecticides. J. Econ. Entomol., (43) : 45-53.

Tanani, M.; Ghoneim, K.; Hamadah, Kh.; Basiouny, A. and Waheeb, H. (2016). Disruptive Effects of Some Novel Chitin Synthesis Inhibitors on the Transaminase Activity in Larval Tissues of Spodoptera littoralis (Lepidoptera: Noctuidae), Inter. J. Res. Stud. Zool., 2(1): 1-12.

Tabashnik, B. E. (1989). Managing resistance with multiple pesticide tactics: theory, evidence, and recommendations. J. Econ. Entomol., 82: 12631269.

Tabashnik, B. E. (1986). Evolution of pesticide resistance in predator/prey systems. Bull. Entomol. Soc. Amer., 32: 156-161.

Temerak, S. A. (2002). Historical records of field cotton leafworm (Spodoptera littoralis) resistance to conventional insecticides as influenced by the resistance programs in Egypt. Resistant Pest Management Newsletter., 12: 7-10.

Tukey, J. (1949). Comparing Individual Means in the Analysis of Variance. Biometrics, 5 (2): 99-114.

Wyatt, G. R. (1967). The biochemistry of sugars and polysaccharides in insects. Adv. Insect Physiol., 4: 287-360.

Younes, M. W. F.; El-Sayed, Y. A. and Hegazy M. M. A. (2008). Effect of Bacillus thuringiensis var Kurstaki on some biocemical paramters of the cotton leafworm, Spodoptera littoralis(Boisd). $4^{\text {th }}$ Int. Conf. Appl. Entomol. Fac. Sci. Cairo Uni., (21-22May, 2008) pp 1-11.

Zidan, Z. H. and Abdel-Megeed M. I. (1988). New approaches in pesticides and insect control. Arabic Publishing House and Delivery Cairo. 605pp (In Arabic Language).

Zohry, N. M. (2006). Aberration of some Insecticides on some biological aspects of the cotton leafworm Spodoptera littoralis (Lepidoptera: Noctuidae). Ph.D. Thesis, Fac. Sci., Ain Shams Univ., Cairo, Egypt.

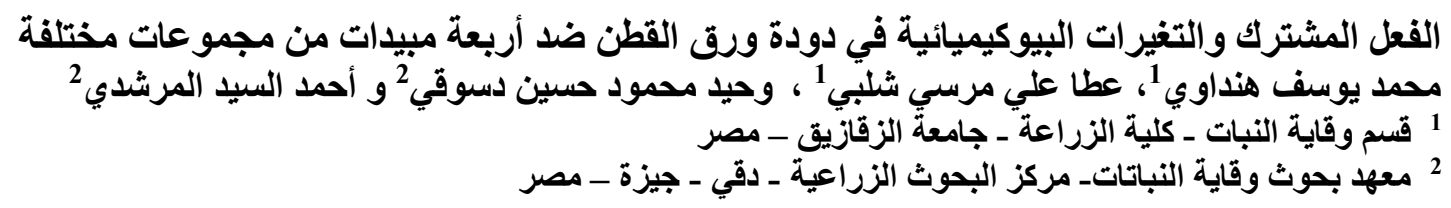

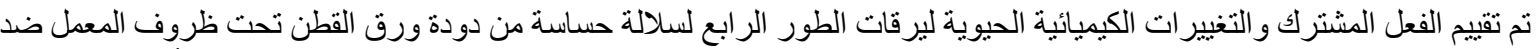

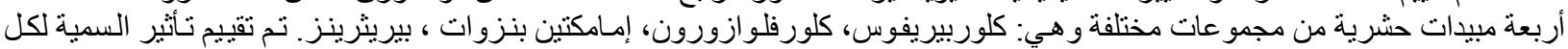

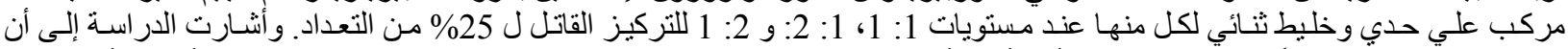

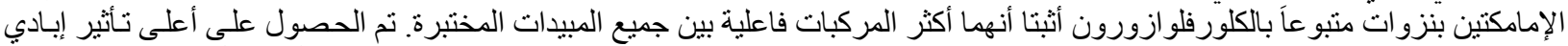

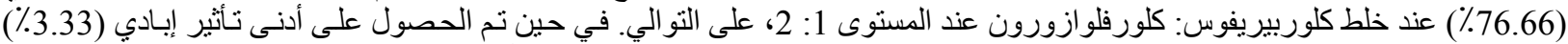

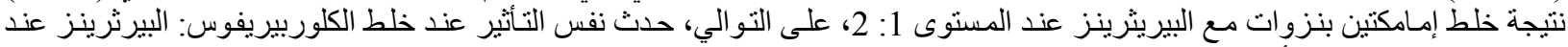

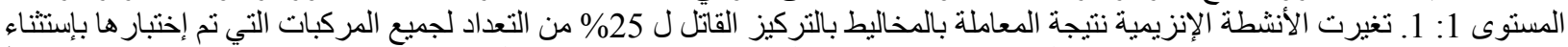

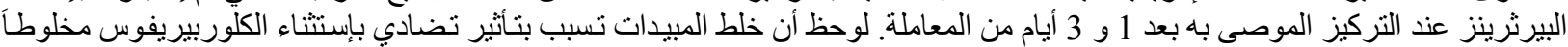

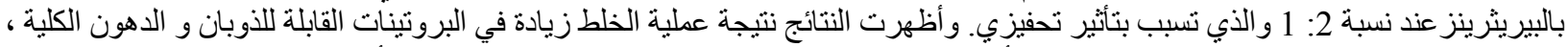

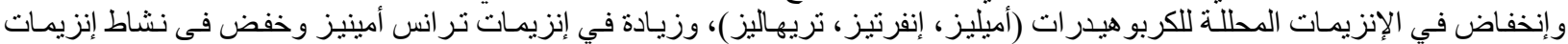

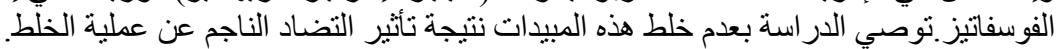


Hendawi, M. Y. et al. 